\title{
Effect of microscopic disorder on magnetic properties of metamaterials
}

\author{
Maxim V. Gorkunov, ${ }^{1,2}$ Sergey A. Gredeskul, ${ }^{1,3}$ Ilya V. Shadrivov, ${ }^{1}$ and Yuri S. Kivshar ${ }^{1}$ \\ ${ }^{1}$ Nonlinear Physics Centre, Research School of Physical Sciences and Engineering, Australian National University, \\ Canberra ACT 0200, Australia \\ ${ }^{2}$ Institute of Crystallography, Russian Academy of Science, Moscow 119333, Russia \\ ${ }^{3}$ Department of Physics, Ben-Gurion University, Beer-Sheva, Israel
}

(Received 10 October 2005; revised manuscript received 10 March 2006; published 16 May 2006)

\begin{abstract}
We analyze the effect of microscopic disorder on the macroscopic properties of composite metamaterials and study how weak statistically independent fluctuations of the parameters of the structure elements can modify their collective magnetic response and left-handed properties. We demonstrate that even a weak microscopic disorder may lead to a substantial modification of the metamaterial magnetic properties, and a $10 \%$ deviation in the parameters of the microscopic resonant elements may lead to a substantial suppression of the wave propagation in a wide frequency range. A noticeable suppression occurs also if more than $10 \%$ of the resonant magnetic elements possess strongly different properties, and in the latter case the defects can create an additional weak resonant line. These results are of a key importance for characterizing and optimizing novel composite metamaterials with the left-handed properties at terahertz and optical frequencies.
\end{abstract}

DOI: 10.1103/PhysRevE.73.056605

PACS number(s): 42.70.Qs, 41.20.Jb, 78.67.Pt

\section{INTRODUCTION}

Artificially fabricated composite conductive structures for electromagnetic waves or metamaterials acquire a growing attention of research during the last years due to their unique properties of negative magnetic permeability and left-handed wave propagation. Being composed of three-dimensional arrays of identical conductive elements, the metamaterials have much in common with conventional optical crystals scaled to support the propagation of microwave or terahertz radiation. In contrast to crystals, metamaterials allow tailoring their macroscopic properties by adjusting the type and geometry of their structural elements. In particular, it is possible to obtain negative permeability in magnetically resonant metamaterials at the frequencies up to hundreds of terahertz $[1,2]$. This appears to be especially useful for the practical realization of the negative refraction phenomenon [3].

The simplest method to create a magnetically resonant metamaterial is to assemble a periodic lattice of the resonant conductive elements (RCEs), where each element is much smaller than the wavelength of the propagating electromagnetic waves, and can be well approximated by a linear LC contour. A small slit provides the contour with a certain capacitance while its shape determines self-inductance. As a result, a resonance of the induced currents and corresponding magnetization resonance occurs. Remarkably, the resistive losses in RCEs are low enough to provide the quality factor of the resonance of the order of $10^{3}$ [1].

During the recent years, a number of ideas have been suggested for achieving better characteristics [4], tunability [5-7], nonlinear wave coupling [8-11] in composite metamaterials by inserting different types of active and passive electronic complements into the resonant circuits. One of the common features of these seemingly different approaches is to modify the macroscopic properties by the identical insertions into a microscopic structure of the metamaterial, assuming technologically ideal fabrication processes. It was always assumed that all RCEs are identical, and no analysis of the effect of random deviations or disorder was carried out. However, one should expect that even weak fluctuations in the microscopic parameters may become critical near the magnetic resonance. On the other hand, after a certain working time, a small amount of RCEs could experience a breakdown and will operate in a way different from that of the majority of the elements. Thus, the problem of disorder appears naturally in the physics of composite metamaterials.

Recent experiments [12] demonstrated a significant decrease of the wave transmission due to defect elements in one-dimensional metamaterial structures. Low transmission in a one-dimensional magnetoinductive waveguide with weak deviations of the element properties was also found in numerical simulations [13]. The first theoretical study of the effects of disorder in three-dimensional metamaterials has been made in Ref. [14], where the magnetic susceptibility for a spatially uniform system [15] has been averaged with respect to random variations of the RCE resonant frequency, and the resulting change of the frequency dispersion of the left-handed composite system has been found analytically.

The method used in Ref. [14] is based on a macroscopic averaging performed prior to a statistical averaging. This is possible only under the assumption that the resonant frequency is a slowly varying random function of the coordinates, and its correlation length $r_{c}$ satisfies the inequality

$$
r_{\text {resp }} \ll r_{c} \ll \lambda,
$$

where $r_{\text {resp }}$ is the characteristic length of the local response (which is usually of the order of a few lattice constants [15]), and $\lambda$ is the wavelength of the electromagnetic wave propagating in the metamaterial. However, the opposite case,

$$
r_{c} \ll r_{\text {resp }},
$$

appears to be more realistic because even the nearest neighboring RCEs are statistically independent. Then the primary 
characteristic of the disordered structure is not the average susceptibility itself but the current distribution, and the magnetic properties of the disordered system are determined by a macroscopic average of the current. If the averaging length is large with respect to the correlation length, i.e., if the inequality (2) is fulfilled, then the macroscopic current is a self-averaged quantity [16], and it should coincide with its ensemble average. In this case, the magnetic properties of the system are described by a statistical mean current.

Below we study systematically the effect of disorder on the averaged characteristics and permeability of metamaterials, and consider two practically important models of the disordered composite metamaterials, assuming the capacitances $C_{\mathbf{n}}$ of different RCEs to be random quantities. In the first model, we assume that the capacitances are completely uncorrelated, but fluctuations are weak. The second model corresponds to the small volume density $\widetilde{n}$ of defect RCEs acting as impurities with substantially different capacitance $\widetilde{C}$. The difference can be very strong here covering two practically important cases of a casual RCE breakdown, $\widetilde{C} \rightarrow \infty$, and the absence of some RCEs, $\widetilde{C} \rightarrow 0$. The impurities make the medium microscopically inhomogeneous. The volume in which the local response is formed has to contain a large number of impurities. In accordance with the condition (2), this implies an additional but not very restrictive condition: the volume density of impurities should not be extremely small, i.e., $\tilde{n} \gg \lambda^{-3}$.

The paper is organized as follows. In Sec. II we analyze the magnetic response of an ideally ordered metamaterial. We start with reminding briefly the method of permeability calculation developed in [15], and also describe a theoretical approach based on the response function of the discrete composite medium, which later allows us to describe the properties and response of rather general microscopically inhomogeneous media. Section III is devoted to the study of effects of small deviations in each resonant element. In Sec. IV, we deal with the case of strong but rarefied impurities. Our general conclusions are accompanied by some specific examples calculated numerically for the typical metamaterial parameters. Finally, in Sec. V we discuss the quality and reliability requirements for the electronic components to be used as RCE insertions in the composite magnetic metamaterials.

\section{RESPONSE OF MAGNETIC METAMATERIALS}

\section{A. Effective permeability}

First we consider a composite metamaterial created by an ideal three-dimensional lattice of identical RCEs. The RCEs are placed in the parallel planes normal to the $z$ axis (see Fig. 1 ). We denote $N$ the total macroscopic number of the lattice sites, and each site is characterized by the index $\mathbf{n}$. We assume that the wavelength of the electromagnetic radiation is much larger than both the RCE size and lattice constant, which allows us to describe the electromagnetic properties of the metamaterial using the effective magnetic permeability and effective dielectric permittivity.

To derive the permeability of the particular metamaterial we apply the procedure developed earlier in Ref. [15]. In brief, the main steps include the following.

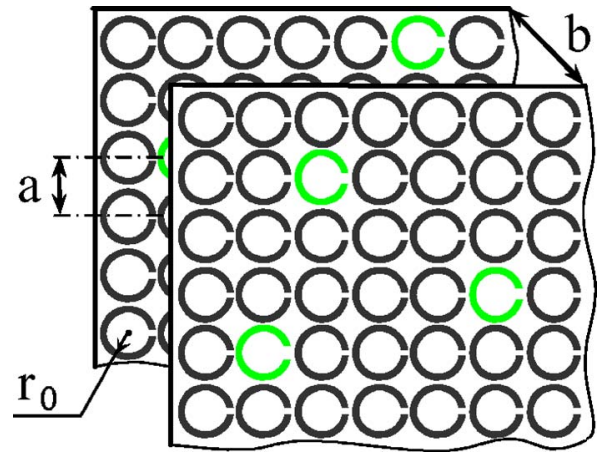

FIG. 1. (Color online) Schematic of two layers of the metamaterial with random insertions of defect resonant elements (shown in green).

(i) The magnetization of the metamaterial exposed to a homogeneous oscillating magnetic field parallel to the $z$ axis is calculated, taking into account the mutual inductive coupling of RCEs.

(ii) The volume averaging over a unit cell of the overall microscopic magnetic field yields the macroscopic magnetic induction.

(iii) Excluding the external field allows us to obtain the relation between the magnetic induction and the macroscopic magnetic field, that defines the permeability.

Below we present these calculations in more detail. The external magnetic field $H_{z}^{(0)}$ oscillating with the frequency $\omega$ gives rise to an electromotive force in each RCE, $\mathcal{E}=i \mu_{0} \omega S H_{z}^{(0)}$, where $S$ is the RCE area. On the other hand, the relation between the external electromotive forces and the induced currents $I_{\mathbf{n}}$ is given by the mutual impedance matrix $[17] \hat{Z}$ with $N^{2}$ elements:

$$
\sum_{\mathbf{m}} Z_{\mathbf{n m}} I_{\mathbf{m}}=\mathcal{E}_{\mathbf{n}},
$$

where for a periodic structure $Z_{\mathbf{n m}}=Z(\mathbf{n}-\mathbf{m})$. The diagonal elements of the matrix $\hat{Z}$ coincide with the RCE selfimpedance:

$$
Z(0)=-i \omega L+\frac{i}{\omega C}+R,
$$

while the nondiagonal elements are determined by the mutual inductance:

$$
Z(\mathbf{n}-\mathbf{m})=-i \omega M(\mathbf{n}-\mathbf{m}) .
$$

In an ideally ordered composite, the currents $I_{\mathbf{n}}$ are the same, and they can be found as

$$
I_{0}=\frac{\mathcal{E}}{Z_{0}}, \quad Z_{0} \equiv \sum_{\mathbf{n}} Z(\mathbf{n}) .
$$

Accordingly, the magnetization of the metamaterial is found as

$$
M_{z}=i \omega \mu_{0} n S^{2} H_{z}^{(0)} Z_{0}^{-1},
$$

where $n$ is the volume density of RCEs. 
Next, we perform the macroscopic averaging of the magnetic field (external plus induced by RCEs). As shown in [15], integrating the field over a unit cell yields a rather general relation, which is independent of the details of the material structure:

$$
B_{z}=\mu_{0}\left(H_{z}^{(0)}+\frac{2}{3} M_{z}\right) .
$$

Combining Eqs. (7) and (8) with the relation $B_{z}=\mu_{0}\left(H_{z}\right.$ $+M_{z}$ ) yields the standard formula of macroscopic electrodynamics $B_{z}=\mu_{0} \mu_{z z} H_{z}$, with the effective permeability of the form

$$
\mu_{z z}=\frac{i Z_{0}-(2 / 3) \omega \mu_{0} n S^{2}}{i Z_{0}+(1 / 3) \omega \mu_{0} n S^{2}} .
$$

Note that the structure of this relation resembles the Clausius-Mossotti formula obtained within the local field theory approximation. As discussed in [15], the permeability (9) reduces to the Clausius-Mossotti relation in the limit of infinitesimally small RCEs. However, for typical metamaterials, this limit is far from being realistic, and we rely on a more general expression for permeability defined by Eq. (9).

\section{B. Metamaterial response function}

Response function $G$ appears to be another useful characteristic of the metamaterial. Mathematically, it is an inverse matrix of the mutual impedance matrix $\hat{G}=\hat{Z}^{-1}$, and it satisfies the equation

$$
\sum_{\mathbf{m}} Z(\mathbf{n}-\mathbf{m}) G(\mathbf{m}-\mathbf{l})=\delta_{\mathbf{n}, \mathbf{l}} .
$$

This matrix has a clear physical meaning. Indeed, according to Eq. (10) it is the distribution of currents for the system with only one RCE (placed at the origin $\mathbf{n}=\mathbf{0}$ ) exposed to the action of the unitary external electromotive force, i.e., $\mathcal{E}_{\mathbf{n}}$ $=\delta_{\mathbf{n}, \mathbf{0}}$. Therefore, $G(\mathbf{n})$ is nothing but the Green's function of the metamaterial.

To find $G$, we solve Eq. (10) via the Fourier transform in the reciprocal space. The latter consists of $N$ wave vectors $\mathbf{k}$ within the first Brillouin zone. Let us define the Fourier transform $\tilde{f}(\mathbf{k})$ of a discrete function $f(\mathbf{n})$ as

$$
\widetilde{f}(\mathbf{k})=\frac{1}{N} \sum_{\mathbf{n}} f(\mathbf{n}) e^{-i \mathbf{k} \cdot \mathbf{n}}
$$

so that the inverse transform has the form

$$
f(\mathbf{n})=\sum_{\mathbf{k}} \tilde{f}(\mathbf{k}) e^{i \mathbf{k} \cdot \mathbf{n}} .
$$

Then the inversion of the difference matrix can be easily performed,

$$
G(\mathbf{n})=\frac{1}{N} \sum_{\mathbf{k}} \frac{e^{i \mathbf{k} \cdot \mathbf{n}}}{\sum_{\mathbf{m}} Z(\mathbf{m}) e^{-i \mathbf{k} \cdot \mathbf{m}}} .
$$

The poles of the Green's function [i.e., zeros of the denominator in Eq. (11)] determine the spectrum of linear waves that can be excited in the composite metamaterial. In the magnetostatic approximation, only a part of the spectrum can be revealed. Conventional relativistic "lightlike" electromagnetic waves remain beyond the validity of this approach. However, as we demonstrate below, small wave vectors and high group velocities of the "lightlike" waves make their contribution negligible. The excitations that determine the Green's function on a microscopic scale can be well explored within the magnetostatic approximation. Being first predicted in Ref. [18], the magnetostatic excitations were observed in ab initio simulations of a metamaterial sample exposed to an external magnetic field [15] and soon after they were explored theoretically in one-, two-, and three-dimensional metamaterial structures in Refs. [13,19,20]. Although the waves are similar in many aspects to quasistatic excitations in magnetic substances (magnetostatic waves and magnons), in metamaterials they are called magnetoinductive waves. In the following we will use this established terminology.

Until now, the spectra of magnetoinductive waves in three-dimensional metamaterials were analyzed only in the nearest and next-nearest neighbor approximations $[13,20]$, which provide only qualitative information. As will be discussed below in Sec. III B, in order to obtain quantitatively reliable results we should take into account hundreds of neighbors.

After changing the summation over the macroscopic number of $\mathbf{k}$ vectors by the integration over the first Brillouin zone, $B_{1}$, we obtain

$$
G(\mathbf{n})=\frac{1}{(2 \pi)^{3} n} \int_{B_{1}} \frac{e^{i \mathbf{k} \cdot \mathbf{n}} d^{3} k}{\sum_{\mathbf{m}} Z(\mathbf{m}) \mathbf{e}^{-i \mathbf{k} \cdot \mathbf{m}}} .
$$

With the help of Eqs. (4) and (5) we can rewrite the denominator to obtain

$$
G(\mathbf{n})=\frac{i \omega C}{(2 \pi)^{3} n} \int_{B_{1}} \frac{\Omega^{2}(\mathbf{k}) e^{i \mathbf{k} \cdot \mathbf{n}} d^{3} k}{\omega^{2}-\Omega^{2}(\mathbf{k})+i \omega \gamma(\mathbf{k})} .
$$

Here the spectrum of magnetoinductive waves, $\Omega(\mathbf{k})$, is in agreement with the results of Ref. [13],

$$
\Omega^{2}(\mathbf{k})=\frac{\omega_{0}^{2}}{1+L^{-1} \sum_{\mathbf{n}} M(\mathbf{n}) e^{i \mathbf{k} \cdot \mathbf{n}}},
$$

and the damping coefficient is determined as

$$
\gamma(\mathbf{k})=\frac{\Omega^{2}(\mathbf{k})}{Q \omega_{0}},
$$

where $\omega_{0}=(L C)^{-1 / 2}$ and $Q=\omega_{0} L / R$ are the RCE resonant frequency and quality factor, respectively.

To isolate the contribution of singular poles, we split the integral in Eq. (13) into the real and imaginary parts,

$$
G(\mathbf{n})=\frac{\omega C}{(2 \pi)^{3} n}[A(\mathbf{n})+i B(\mathbf{n})],
$$

where the imaginary part allows a straightforward numerical integration, 


$$
B(\mathbf{n})=\int_{B_{1}} \frac{\Omega^{2}(\mathbf{k})\left[\omega^{2}-\Omega^{2}(\mathbf{k})\right] e^{i \mathbf{k} \cdot \mathbf{n}}}{\left[\omega^{2}-\Omega^{2}(\mathbf{k})\right]^{2}+\omega^{2} \gamma^{2}(\mathbf{k})} d^{3} k,
$$

whereas the real part

$$
A(\mathbf{n})=\int_{B_{1}} \frac{\omega \Omega^{2}(\mathbf{k}) \gamma(\mathbf{k}) e^{i \mathbf{k} \cdot \mathbf{n}}}{\left[\omega^{2}-\Omega^{2}(\mathbf{k})\right]^{2}+\omega^{2} \gamma^{2}(\mathbf{k})} d^{3} k
$$

acquires a contribution at the surface $S_{\mathbf{K}}$ built by the magnetoinductive wave vectors $\mathbf{K}(\omega)$, obeying the relation $\Omega[\mathbf{K}(\omega)]=\omega$. Since the RCE quality factor is high, the singular part dominates in Eq. (18). In the vicinity of the surface $S_{\mathbf{K}}$, we can write $\Omega(\mathbf{k}) \simeq \omega+\mathbf{k} \cdot \mathbf{v}(\mathbf{K})$, where $\mathbf{v}(\mathbf{K})$ stands for the group velocity. Next, we integrate along the surface normal reducing the volume integration in Eq. (18) to the surface integral over $S_{\mathbf{K}}$,

$$
A(\mathbf{n})=\pi \omega \int_{S_{\mathbf{K}}} \frac{e^{i \mathbf{K} \cdot \mathbf{n}} d^{2} K}{v(\mathbf{K})},
$$

which is more suitable for numerical calculations.

Analyzing Eqs. (17) and (19), it is easy to conclude that accounting for the "lightlike" modes would not lead to any noticeable corrections. These modes are located in the center of the Brillouin zone near the point $\mathbf{k}=0$, and their contribution to the integral $B$ is apparently small. On the other hand, the light group velocity is about two orders of magnitude higher than that of the magnetoinductive waves. Therefore, the "lightlike" part in $A$ is also negligible.

\section{WEAK FLUCTUATIONS}

\section{A. Magnetic permeability of disordered structures}

In order to model the effect of a weak disorder in the composite media, we assume that the values of the RCE self-impedances experience random uncorrelated deviations due to the capacitance fluctuations. This may correspond to the results of a real fabrication process when the capacitances of different resonators are not identical, and they can be treated as independent quantities. In this case, the correlation radius coincides with the lattice constant, $r_{c}=a$, and the inequality (2) is satisfied. Obviously, strong uncertainty should totally destroy the macroscopic metamaterial response. We assume here that the fluctuations are weak and study their effect on the permeability in the second order of the perturbation theory with respect to the capacitance fluctuations, using the methods known in the solid state physics [21].

We define the local fluctuations of the RCE impedance as

$$
\delta_{\mathbf{n}}=\frac{1}{\omega}\left(\frac{1}{C_{\mathbf{n}}}-\frac{1}{C}\right),
$$

where

$$
\frac{1}{C} \equiv\left\langle\frac{1}{C_{\mathrm{n}}}\right\rangle,
$$

and the angle brackets stand for the statistical averaging.

To calculate the magnetic permeability of a disordered metamaterial, we turn again to the composite medium ex- posed to a homogeneous external radiation. Equation (3) now takes the form

$$
\sum_{\mathbf{m}} Z(\mathbf{n}-\mathbf{m}) I_{\mathbf{m}}+i \delta_{\mathbf{n}} I_{\mathbf{n}}=\mathcal{E} .
$$

Applying an iterating procedure to Eq. (22), we obtain

$$
I_{\mathbf{n}}=I_{0}-i \sum_{\mathbf{m}} G(\mathbf{n}-\mathbf{m}) \delta_{\mathbf{m}} I_{0}-\sum_{\mathbf{m}, \mathbf{p}} G(\mathbf{n}-\mathbf{m}) G(\mathbf{m}-\mathbf{p}) \delta_{\mathbf{m}} \delta_{\mathbf{p}} I_{\mathbf{p}} .
$$

The assumption of weak fluctuations allows us to substitute $I_{0}$ instead of $I_{\mathbf{p}}$ into the last term of Eq. (23).

The important step in our subsequent analysis is the macroscopic averaging of this equation. The size of the (macroscopic) volume of averaging should be small with respect to the wavelength of the external field. On the other hand, this size is much larger than the radius of the capacitance correlations. Therefore, from the statistical point of view, the volume of averaging can be considered infinite. As a result, instead of the volume averaging the statistical averaging can be performed [16]. Taking into account that $\left\langle\delta_{\mathbf{n}}\right\rangle=0$, and the capacitances of different RCEs are statistically independent, $\left\langle\delta_{\mathbf{m}} \delta_{\mathbf{p}}\right\rangle \propto \delta_{\mathbf{m}, \mathbf{p}}$ we obtain that in the second order of the perturbation theory the average current induced in RCEs can be presented as [cf. Eq. (6)]

$$
\left\langle I_{\mathbf{n}}\right\rangle=\frac{\mathcal{E}}{Z_{\mathrm{eff}}},
$$

where the effective impedance

$$
Z_{\text {eff }}=Z_{0}+\delta^{2} G(\mathbf{0})
$$

involves the square of the standard deviation,

$$
\delta^{2}=\left\langle\delta_{\mathbf{n}}^{2}\right\rangle .
$$

Accordingly, the permeability of the weakly disordered metamaterial takes the form (9) with the impedance $Z_{0}$ being replaced by the effective impedance $Z_{\text {eff }}$.

We note that the fluctuations contribute to both imaginary and real parts of $Z_{\text {eff }}$. The imaginary part of the correction is determined by $B(\mathbf{0})$ and it affects mainly the real part of the permeability. The real part of the correction leads to an increase of $\operatorname{Im}\left(\mu_{z z}\right)$, and it enhances the effective dissipation. Remarkably, this occurs even when RCE losses are absent, and the energy is dissipated without conversion into heating, i.e., this dissipation mechanism is analogous to the Landau damping. The nature of this nonheating dissipation becomes clear if we note that it is determined by the term $A(\mathbf{0})$ that combines the contributions from all magnetostatic waves excited at the given frequency $\omega$. This suggests that the incident radiation experiences scattering on microscopic fluctuations. The similar effect arising in electromagnetic media without positional order (see, e.g., Ref. [22] and references therein) is known as scattering losses or Raleigh scattering. In our case, a very small correlation radius suppresses the scattering into "lightlike" modes, but the scattering into magnetoinductive waves remains strong. 


\section{B. Weak disorder in a typical metamaterial}

As a specific example of the application of our theory, we calculate the averaged magnetic permittivity numerically for typical metamaterial parameters with a weak disorder. We assume circular RCEs with radius $r_{0}=2 \mathrm{~mm}$, wire thickness $l=0.1 \mathrm{~mm}$, which leads to the self-inductance $L=8.36 \mathrm{nHn}$ (see Ref. [17]). To obtain RCEs with the resonant frequency $\omega_{0}=6 \pi \times 10^{9} \mathrm{rad} / \mathrm{s}\left(\nu_{0}=3 \mathrm{GHz}\right)$, we take $C=0.34 \mathrm{pF}$. The lattice constants are $a=2.1 r_{0}$ in the plane and $b=r_{0}$ in the $z$ direction. The RCE quality factor $Q$ can reach the values of $10^{3}[1]$. However, we expect that the insertion of diodes or other electronic components can lower this value to $Q=10^{2}$.

First, we calculate the linear spectrum of magnetoinductive waves from Eq. (14) and find a strong evidence of the long-range interaction effects: the lattice sum converges rather slow. In particular, in order to get an accuracy of a few percent, we have to expand the summation radius to at least ten lattice constants. A further increase of the summation limits would be unjustified, since the sum should be calculated over the distances much smaller than the radiation wavelength. We believe that the problem of the exact calculation of the spectrum of magnetoinductive waves in the three-dimensional case needs a separate detailed consideration. On the other hand, we observe that the maximum uncertainty in the spectrum takes place along specific directions perpendicular to the edges of the Brillouin zone. Therefore, the resulting error in the integrals determining the discrete Green's function is extremely small.

Evaluating numerically the integrals $A(\mathbf{0})$ and $B(\mathbf{0})$ according to Eqs. (17) and (19), we obtain the effective impedance and corresponding magnetic permeability. The frequency dispersion of the permeability $\mu_{z z}$ is presented in Fig. 2 for the unperturbed metamaterial and for several values of the standard deviation. Apparently, already $10 \%$ uncertainty changes dramatically the permeability frequency dispersion near the resonance. The effect is most pronounced for the frequencies below the resonance. In a wide range, the imaginary part of $\mu$ becomes comparable with the real part, and a weakly disordered nondissipative medium acts as strongly dissipative. In the range of negative $\mu$ above the resonance, the losses are also considerably higher than those in a perfect metamaterial. As a result, the frequency range appropriate for the negative refraction shrinks.

\section{RAREFIED STRONG DEFECTS}

\section{A. Concentration expansion}

In this section we consider a metamaterial with a small amount of randomly distributed defective RCEs that differ strongly from the regular RCEs, and therefore can be treated as impurities. We assume that the dimensionless concentration of such impurities $c=\widetilde{n} / n$ is low, i.e., $c \ll 1$. As before, we assume that the deviations from the structure parameters appear due to a difference in the RCE capacitances, and the capacitance of the impurity, $\widetilde{C}$, can even become very large corresponding to a casual breakdown. We neglect any correlation between the impurities, but assume that two impurities cannot be placed on the same site. The applicability condi-
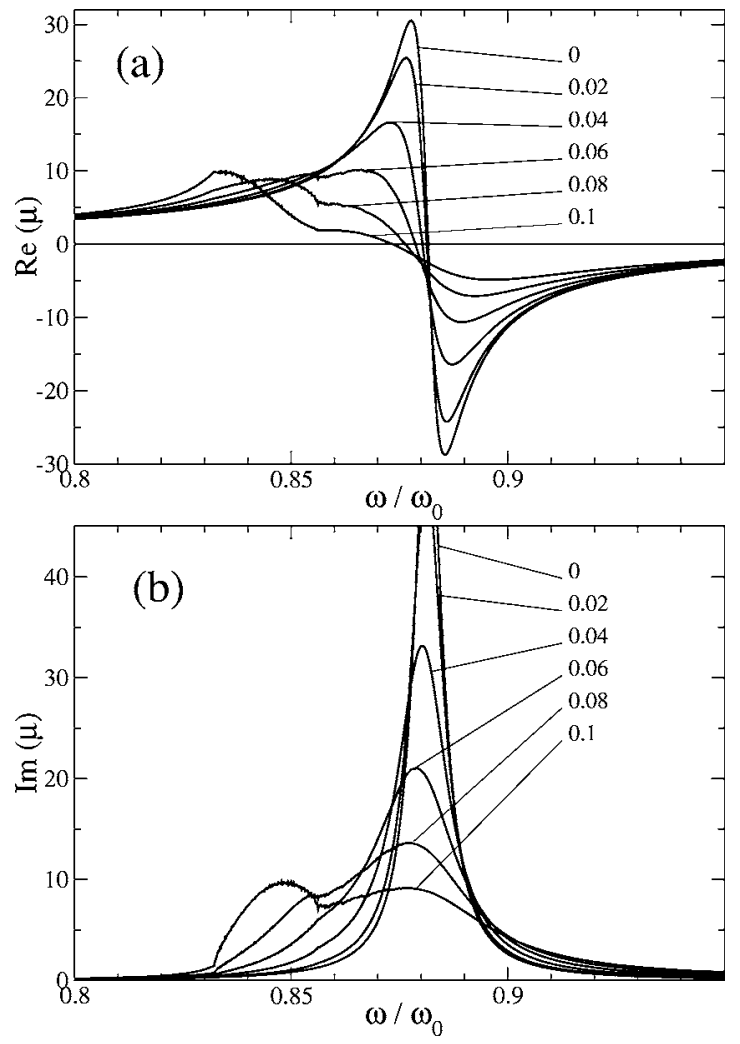

FIG. 2. Real (a) and imaginary (b) parts of the magnetic permeability of metamaterial without fluctuations and with weak capacitance fluctuations, $\delta \omega_{0} C$, marked on the plots.

tion, $\tilde{n} \gg \lambda^{-3}$, defines the lower limit for the impurity concentration, $c_{\min } \sim 10^{-3}$ for a typical metamaterial. Above this limit, we can calculate statistically averaged current and construct its concentration expansion using standard techniques $[16,23]$.

In the system with impurities, the microscopic current distribution is substantially inhomogeneous. We are interested in the normalized averaged value, defined as

$$
\langle I\rangle=\frac{1}{N} \sum_{\mathbf{n}} I_{\mathbf{n}}
$$

where the summation is performed over a volume that includes a macroscopic number of impurities. The concentration expansion of the averaged current can be written in the following form $[16,23]$ :

$$
\begin{aligned}
\langle I\rangle= & I_{0}+c \sum_{\mathbf{p}_{1}}\left[I_{1}\left(\mathbf{p}_{1}\right)-I_{0}\right] \\
& +\frac{c^{2}}{2} \sum_{\mathbf{p}_{1} \neq \mathbf{p}_{2}}\left[I_{2}\left(\mathbf{p}_{1}, \mathbf{p}_{2}\right)-I_{1}\left(\mathbf{p}_{1}\right)-I_{1}\left(\mathbf{p}_{2}\right)+I_{0}\right]+\ldots,
\end{aligned}
$$

where 


$$
I_{1}\left(\mathbf{p}_{1}\right) \equiv \frac{1}{N} \sum_{\mathbf{n}} I_{\mathbf{n}}^{(1)}\left(\mathbf{p}_{1}\right)
$$

describes the averaged current when a single impurity is located at the site $\mathbf{p}_{1}$, whereas the function

$$
I_{2}\left(\mathbf{p}_{1}, \mathbf{p}_{2}\right) \equiv \frac{1}{N} \sum_{\mathbf{n}} I_{\mathbf{n}}^{(2)}\left(\mathbf{p}_{1}, \mathbf{p}_{2}\right)
$$

describes the same value for two impurities placed at the sites $\mathbf{p}_{1}$ and $\mathbf{p}_{2}$. The higher-order terms involve the averaged solutions for more impurities. Clearly, we can write formally the expressions for any term of the expansion [23]. However, in this paper we focus on the strongest effects that are linear in the impurity concentration. To calculate the corresponding coefficient, first we should find the corresponding current distribution.

In an impure system exposed to a homogeneous external field, the impedance matrix equation (3) takes the form

$$
\sum_{\mathbf{m}} Z(\mathbf{n}-\mathbf{m}) I_{\mathbf{m}}+i \Delta \sum_{\mathbf{p}} \delta_{\mathbf{n}, \mathbf{p}} I_{\mathbf{p}}=\mathcal{E}
$$

where the summation in the second term on the lhs is performed over the impurity sites $\mathbf{p}$ only, and

$$
\Delta=\frac{1}{\omega}\left(\frac{1}{\tilde{C}}-\frac{1}{C}\right) .
$$

Inverting the matrix $\hat{Z}$, we rewrite Eq. (30) as follows:

$$
I_{\mathbf{n}}=I_{0}-i \Delta \sum_{\mathbf{p}} G(\mathbf{n}-\mathbf{p}) I_{\mathbf{p}} .
$$

If a single impurity is located at the site $\mathbf{p}_{1}$, Eq. (32) taken at $\mathbf{n}=\mathbf{p}_{1}$ yields

$$
I_{\mathbf{p}_{1}}=\frac{I_{0}}{1+i \Delta G(\mathbf{0})},
$$

and the current distribution in this case becomes

$$
I_{\mathbf{n}}^{(1)}\left(\mathbf{p}_{1}\right)=I_{0}\left(1-\frac{i \Delta G\left(\mathbf{n}-\mathbf{p}_{1}\right)}{1+i \Delta G(\mathbf{0})}\right) .
$$

Averaging this expression according to Eq. (29) and substituting the result into Eq. (28) leads to the same results (24) and (9) for the averaged current and effective permeability correspondingly, with the effective impedance in the form

$$
Z_{\mathrm{eff}}=Z_{0}+c \frac{i \Delta}{1+i \Delta G(\mathbf{0})} .
$$

\section{B. Effective magnetic permeability}

To support our results by a specific example, we take typical parameters of metamaterials used above in Sec. III B. First, we study the effect of impurities with an infinite capacitance, $\widetilde{C} \rightarrow \infty$, which corresponds, for instance, to a casual breakdown of the varactor diode insertions. Next, we deal with other impurities that either do not contribute to the magnetization or are just absent; this latter case is modeled
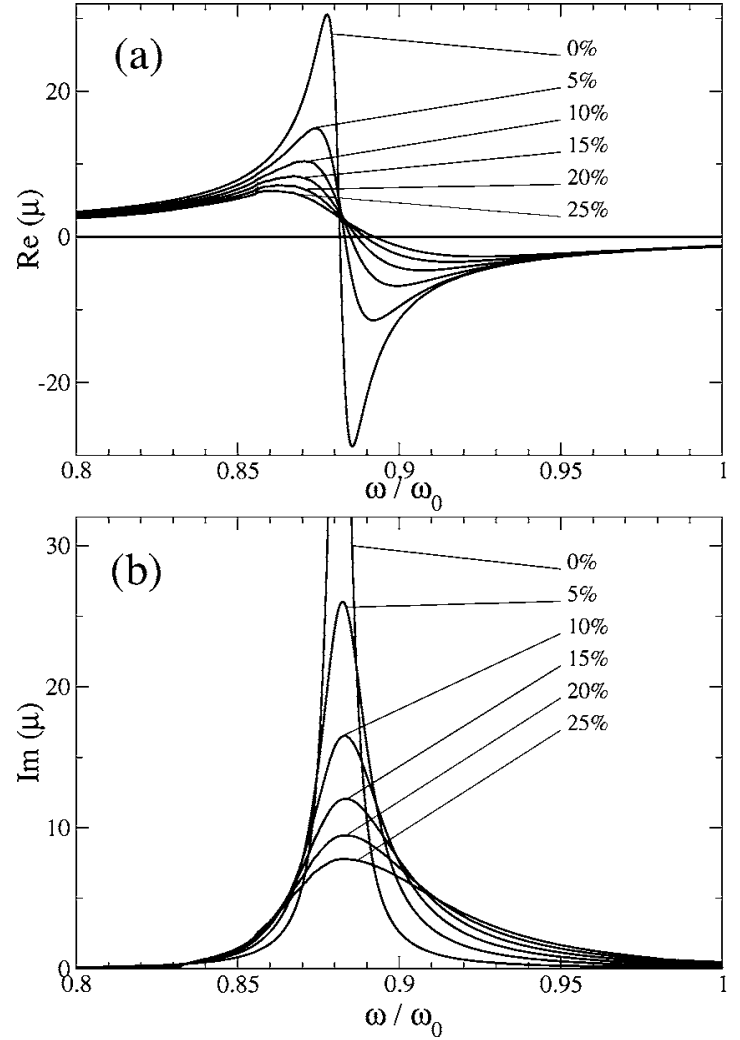

FIG. 3. (a) Real and (b) imaginary parts of the magnetic permeability of metamaterials without and with impurities (the impurity concentration is shown near the curves).

by setting $\widetilde{C} \rightarrow 0$. Although these cases are two opposite extreme limits, the resulting averaged permeabilities look similar and differ only by a few percent. This result is not surprising because in both cases the currents induced in the defect RCEs are either small or vanish.

In Fig. 3 we present the real and imaginary parts of the magnetic permeability for several concentrations of the missing RCEs. As follows from those results, the resonance is attenuated much more than one could expect from the fact that a small part of RCEs do not contribute to the resonance. The reason for such an enhancement is the scattering losses that reduce the effective quality factor by an order of magnitude or more. We note also that the metamaterial is more sensitive to such perturbations in the frequency range of negative $\mu$. The composite medium can tolerate $5 \%$ or less malfunctioning RCEs, but for $10 \%$ and more a substantial damping of the resonance occurs, and the imaginary part of the permeability becomes comparable with its real part.

The impurities with a finite resonant frequency, i.e., $\widetilde{\omega}_{0} \neq 0$, which strongly differs from the main frequency $\omega_{0}$, cause the observable effects, even if their concentration is extremely low. As seen in Fig. 4, only $1 \%$ of such impurities can build up their own weak resonance, with the position of the resonance shifted from the impurity eigenfrequency.

In the linear approximation used here, the effect of mutual interaction of impurities is neglected. Therefore, we do not account for magnetoinductive waves arising at the frequencies close to the impurity resonance. We expect that the cor- 


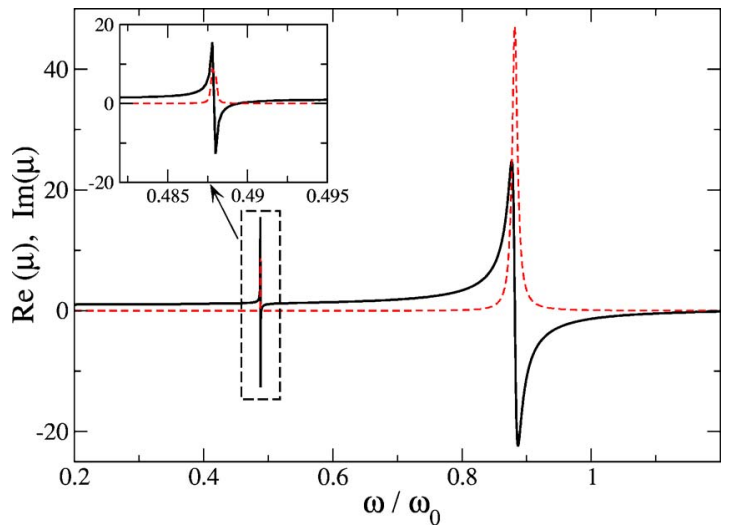

FIG. 4. (Color online) Real (solid) and imaginary (dashed) parts of the permeability of metamaterial with $1 \%$ of randomly distributed defects with the eigenfrequency $\widetilde{\omega}_{0}=0.5 \omega_{0}$. The inset shows a blowup of the impurity resonance.

responding scattering losses will broaden the resonance. However, the detailed analysis of this and related phenomena is beyond the scope of this paper.

\section{CONCLUDING REMARKS}

We have demonstrated that a weak microscopic disorder in the conducting elements of composite metamaterials can lead to a substantial modification of their resonant magnetic response. According to our results, already $10 \%$ disorder in the parameters causes substantial scattering of the incident radiation into magnetoinductive waves. This modifies strongly the macroscopic permeability of the metamaterial, leading to an increase in losses. We believe that the study of the effect of disorder and disorder-induced losses are of critical importance for engineering novel metamaterials with various electronic elements inserted into their microscopic resonators. The inserted elements should possess no more than a few percent deviation of their capacitance and/or inductance to ensure that the metamaterial properties are not distorted dramatically. Another restriction concerns the insertion stability. The results obtained above demonstrate that the metamaterial tolerates no more than $10 \%$ of defect RCEs. Casual breakdown of a larger number of the elements causes strong damping of the wave propagation in metamaterials.

Our results show that even a small amount of defects can build up a noticeable additional magnetic resonance, and it can be useful for simple methods of sensitive quality control of metamaterials. If RCE contains not a single capacitive element but a combination of insertions with an ordinal slit, a casual insertion breakdown would switch RCE to another resonant frequency. The corresponding narrow gap (or peak) in the metamaterial transmission can be used as a sensitive measure of the concentration of damaged RCEs.

Finally, we point out that the effects of a microscopic disorder discussed here would be crucially important in the nanostructured metamaterials, even with simple RCEs without electronic components. Clearly, the fabrication of resonant elements on such scales is less accurate, so that random fluctuations of the RCE shapes can lead to deviations of the self-inductance and capacitance. Disorder in the positions of RCEs determines the deviations of the mutual inductance. We expect that the results obtained here can be important for qualitative estimates of the properties of nanostructured lefthanded media. However, the problem requires more systematic analysis.

\section{ACKNOWLEDGMENTS}

This work was supported by the Australian Research Council and the Israel Science Foundation. M.G. and S.G. thank the Nonlinear Physics Centre of the Research School of Physical Sciences and Engineering at the Australian National University for warm hospitality during their stay in Canberra and financial support.
[1] R. A. Shelby, D. R. Smith, and S. Schultz, Science 292, 77 (2001).

[2] S. Linden, C. Enkrich, M. Wegener, J. F. Zhou, T. Koschny, and C. M. Soukoulis, Science 306, 1351 (2004).

[3] J. B. Pendry, Phys. Rev. Lett. 85, 3966 (2000).

[4] S. A. Tretyakov, Microwave Opt. Technol. Lett. 31, 163 (2001).

[5] M. Gorkunov and M. Lapine, Phys. Rev. B 70, 235109 (2004).

[6] S. O'Brien, D. McPeake, S. A. Ramakrishna, and J. B. Pendry, Phys. Rev. B 69, 241101(R) (2004).

[7] O. Reynet and O. Acher, Appl. Phys. Lett. 84, 1198 (2004).

[8] M. Lapine, M. Gorkunov, and K. H. Ringhofer, Phys. Rev. E 67, 065601(R) (2003).

[9] A. A. Zharov, I. V. Shadrivov, and Yu. S. Kivshar, Phys. Rev. Lett. 91, 037401 (2003).

[10] M. Lapine and M. Gorkunov, Phys. Rev. E 70, 066601 (2004).

[11] M. V. Gorkunov, I. V. Shadrivov, and Yu. S. Kivshar, Appl.
Phys. Lett. 88, 071912 (2006).

[12] X. P. Zhao, Q. Zhao, L. Kang, J. Song, and Q. H. Fu, Phys. Lett. A 346, 87 (2005).

[13] E. Shamonina, V. A. Kalinin, K. H. Ringhofer, and L. Solymar, J. Appl. Phys. 92, 6252 (2002).

[14] A. A. Zharov, I. V. Shadrivov, and Yu. S. Kivshar, J. Appl. Phys. 97, 113906 (2005).

[15] M. Gorkunov, M. Lapine, E. Shamonina, and K. H. Ringhofer, Eur. Phys. J. B 28, 263 (2002).

[16] I. M. Lifshits, S. A. Gredeskul, and L. A. Pastur, Introduction to the Theory of Disordered Systems (Wiley, New York, 1989).

[17] L. D. Landau and E. M. Lifschitz, Electrodynamics of Continuous Media (Pergamon, Oxford, 1984).

[18] E. Shamonina, V. A. Kalinin, K. H. Ringhofer, and L. Solymar, Electron. Lett. 38, 371 (2002).

[19] M. C. K. Wiltshire, E. Shamonina, I. R. Young, and L. Soly 
mar, Electron. Lett. 39, 215 (2003).

[20] I. V. Shadrivov, A. A. Zharov, N. A. Zharova, and Yu. S. Kivshar, Photonics Nanostruct. Fundam. Appl. 4, 69 (2006).

[21] I. M. Lifshitz and L. N. Rosentsveig, Zh. Eksp. Teor. Fiz. 16,
967 (1946).

[22] S. Tretyakov, Analytical Modelling in Applied Electromagnetics (Artech House, Boston, 2003).

[23] I. M. Lifshits, Nuovo Cimento, Suppl. 3, 716 (1956). 